Document downloaded from:

http://hdl.handle.net/10251/35747

This paper must be cited as:

Santos, RS.; Borges, MRS.; Canos Cerda, JH.; Gomes, JO. (2011). The assessment of information technology maturity in emergency response organizations. Group Decision and Negotiation. 20(5):593-613. doi:10.1007/s10726-011-9232-z.

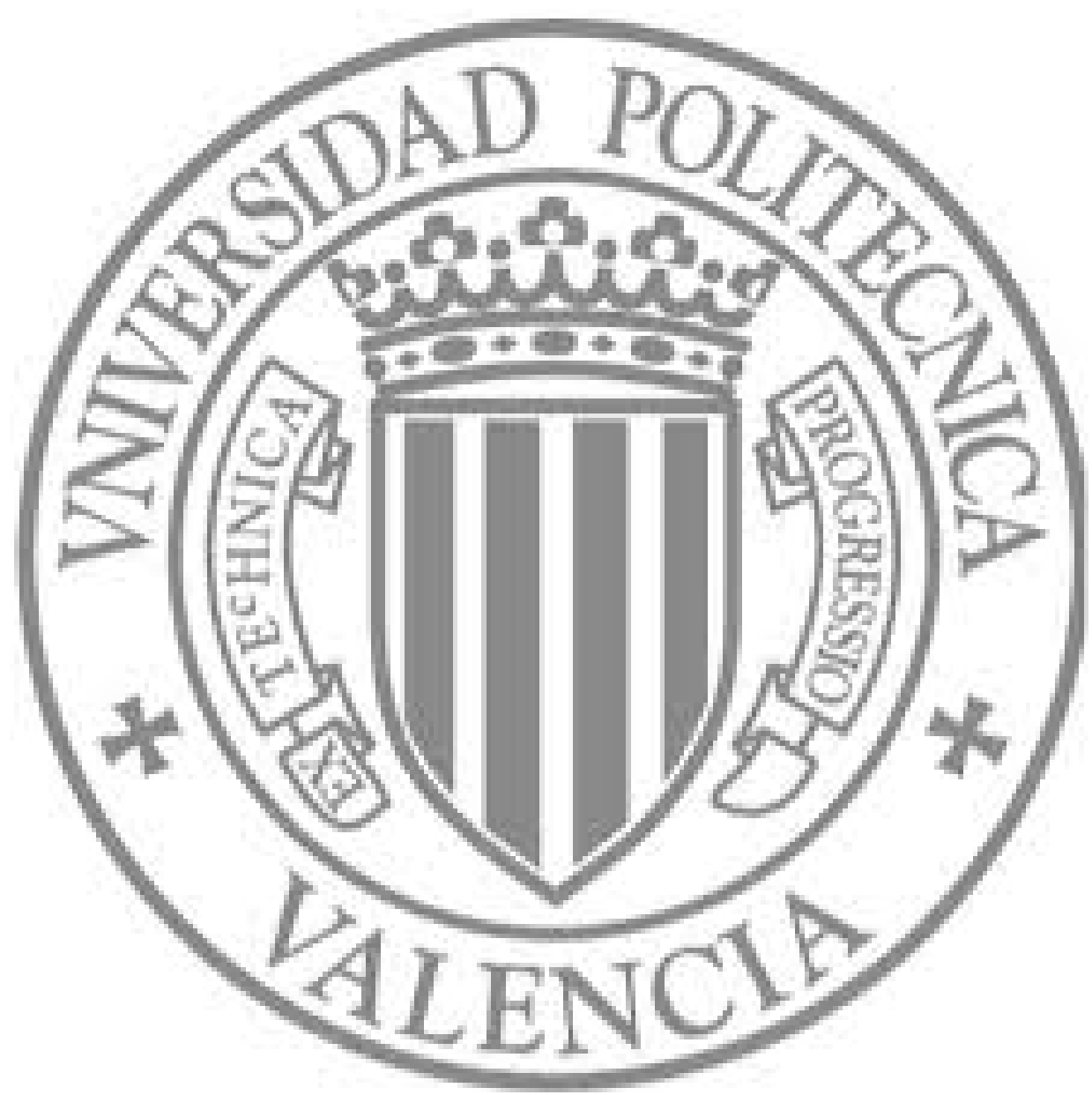

The final publication is available at

http://dx.doi.org/10.1007/s10726-011-9232-z

Copyright

INFORMS (Institute for Operations Research and Management Sciences) 


\title{
The Assessment of Information Technology Maturity in Emergency Response Organizations
}

\author{
Raphael S. Santos ${ }^{1}$, Marcos R. S. Borges ${ }^{1}$, Jose H. Canós ${ }^{2}$ José Orlando Gomes ${ }^{1,3}$ \\ ${ }^{1}$ Graduate Program in Informatics - IM \& NCE Federal University of Rio de \\ Janeiro, Brazil \\ raphaels@posgrad.nce.ufrj.br, \{mborges, joseorlando\}@nce.ufrj.br \\ ${ }^{2}$ Dept. of Computer Science, Technical University of Valencia, Spain \\ jhcanos@dsic.upv.es \\ ${ }^{3}$ Industrial Engineering Department
}

\begin{abstract}
In emergency response organizations, information technologies are not adequately explored. Sometimes, the mere adoption of new information technologies is not productive, as their efficient use depends on other interrelated technologies and the environment where they are installed. This work describes a model to help organizations understand their capability in respect to the adoption of these technologies. The model also helps the performing of the evaluation from different perspectives, making it suitable to collaborative evaluation. Using the proposed model, an organization can measure its maturity level in different aspects of the evaluation and guide the investment on its capabilities. Part of the model has been developed for emergency response organizations and the information technology dimension of the model has been applied to two fire department installations.
\end{abstract}

Keywords: collaborative assessment, maturity model, investment decisions.

\section{Introduction}

The relevance and need of good emergency management are gaining greater evidence in the most varied sectors of society. And, simultaneously, complexity of emergencies is increasing due to many reasons. For instance, emergencies affecting big areas may put in risk a large number of people and properties. This complexity makes the interaction between the various organizations involved, as well as the systematic and organized management of emergencies, essential requirements. to reduce their consequences (Turoff et al. 2004a).

Emergency management can be divided into four stages that cover the full course of an emergency: mitigation, preparation, response, and recovery (Lindel et al. 2007). Among them, the response phase is possibly the most complex. Response actions are usually carried out by several teams which should work in a manner as cooperative and articulated as possible to eliminate or reduce the impact of the disaster. These teams usually follow established procedures to deal with emergencies contained in emergency plans. In most events, actions are coordinated centrally but decisions are made at both central and local levels. Information plays an important role in these decisions.

Emergency response has a high degree of dynamism and uncertainty, demanding speed in the actions realized and not tolerating faults. The dynamic and uncertain nature of the response prevent the complete definition of the actions to be 
performed, the time they will take place, the resources needed and their performers (Turoff 2002). The complexity may be augmented as the professional involved often execute their tasks

When things go wrong in emergency management, the reasons are generally related to breakdowns in information, communication, and/or coordination (Dykstra 2003).The problem of lacking information may be mitigated, or even solved, through the use of information technologies. These technologies play a fundamental role in emergency responses, helping decision makers as well as action performers. However, information technologies are not adequately exploited by emergency response organizations, especially those with limited resources. This situation is observed principally in developing countries, where these organizations often do not have adequate resources. In these organizations, just adopting information technologies is not productive. Rather, a previous understanding of the relationship with other technologies and resources may better direct new investments.

In this work, we describe a model and a method to assist in understanding this relationship and, as a consequence, to guide the investments relative to the response activities of emergency organizations. In this way, we hope it will be possible to increase these organizations' response capacity. Such a complex evaluation requires specialists from different backgrounds and expertise, who should integrate their views to portray the organization's technology maturity. The model and the method were designed in such way to make them appropriate to be used by groups of experts working collaboratively.

The proposed model uses some concepts borrowed from maturity models used to assess organizations in various domains (King and Teo 1997; Paulk et al. 1995). Specifically, it consists of levels composed of several variables. These levels are organized according to the complexity of assessing the organization relative to those variables. The higher levels, related to emergency response dimensions such as collaboration, communication, Information Management, and others, are decomposed until a level is reached whose variables can be easily measured through analysis or observation.

Once applied, the model assesses the emergency organization's response capability along the different dimensions, determining its maturity in the response activities. After the evaluation, the model allows the organization to see its capability related to response actions. From this visualization the organization identifies its positive and negative points, and can thereby plan possible improvements to increase its response capacity.

We applied the model in several units of a fire-fighting organization. To illustrate the process, we have focused in the Information Management dimension, since accessing to the right information is a key requirement of any emergency response process. We describe how we instantiated the model to this organization, and show the results of the evaluation of two of the units.

This paper is structured as follows. Section 2 sets the background for the remainder of the paper. Specifically, we describe the domain of emergency response, focusing on its information management requirements; also, we describe the main features of Emergency Response Information Systems, and raise the problem of the evaluation of the emergency response capability of organizations with regard to the Information Technologies, which is the main subject of the paper. In Section 3, we propose a maturity-based model to assess 
such capability; the application of the model is guided by a method which is also described in detail. We have applied the model and the method to a fire-fighting organization of the State of Rio de Janeiro; the application is described in Section 4. Finally, Section 5 concludes the paper.

\section{Background}

An emergency begins when a dangerous situation requiring immediate action happens and ends when that situation is resolved (Diniz et al. 2008). The (usually short) time interval within which activities to reduce the effects of the undesirable event are performed is called the emergency response.

The main objectives of emergency responses are saving lives, stabilizing the incident, and preserving property and infrastructure (Bigley and Roberts 2001). Responses are complex and do not tolerate failures, as these may have serious consequences. A successful response requires that one or more teams, from one or more organizations (fire departments, police, medical organizations, civil defense, public agencies, etc.), interact satisfactorily. These teams must possess an adequate level of preparation as they always operate under the pressures characteristic of this phase: they never can fail and must act rapidly, often without the information necessary for this (FEMA 1998).

Thus, the success of a response operation depends on the collaboration and coordination among the teams (and their members) involved. A possible way of analyzing a response organization is measuring how experienced their members are in coordinating themselves. This coordination capability comes from both training and previous experiences. Matured organizations, i.e., those that participated in several emergencies, are expected to respond more effectively to emergency situations.

The success also depends on equipments available, on infrastructure and particularly on the appropriate level of relevant information. Information technologies play an important role in today's emergency situations and special attention has been given to this issue by many studies on emergency response (Turoff 2002)

\subsection{Information Management in Emergency Response}

There are several important aspects related to emergency response. As mentioned, a response is the result of the coordinated activities of a number of responders. The coordination is defined in the emergency response plan (or emergency plan), which also includes the information needed in the different emergency scenarios (see below). Responders can be members of the in-place response teams, or be participating in the decision making processes at the control room. In both cases, most decisions will be made collaboratively, based on the expertise of different people working in either places.

Knowledge management is key to make a decision (Gu and Mendonça 2005). As stated by Hale "...the key obstacle to effective crisis response is the communication needed to access relevant data or expertise and to piece together an accurate understandable picture of reality" (Hale, 1997). In emergency settings, there are three sources of knowledge that are combined to be used by decision makers (Diniz et al. 2008). Figure 1 illustrates this concept. First, the Previous Personal Knowledge (PPK) is embedded in each emergency responder's 
mind. It has been acquired during past experiences, training, and simulations of real-life settings. This type of knowledge is fundamental in this domain because it reduces the time needed to make decisions. It is tacit, highly personal, and hard to formalize (Nonaka and Takeuchi 1995). In some way, the amount of PPK of the team may express the level of preparedness of the personnel involved.

Second, the Previous Formal Knowledge (PFK) is usually explicit and does not change during the course of the emergency. One of its main sources is the emergency plan, which includes the information needed to perform the different response actions, such as local maps, description of the infrastructures, and so forth. The amount and usefulness of PFK available to an emergency team can also define the capability of the team in dealing with emergency situations of the kind.

The third key source of knowledge in emergencies is that originating from developments in the emergency settings, called Current Contextual Knowledge (CCK). It is essential for the quality of decisions in the dynamic emergency environments. Examples of CCK are temperature measures captured by a sensor, aerial images about the incident or information from human sources expressed by sentences such as: "There are two people inside the building, one on the tenth floor and the other on the fifth," "this building will collapse soon". We can identify two types of CCK, one corresponding to the development of the emergency, and the other one encompassing the actions performed by the emergency teams. In both cases, the information has to be perceived and processed in order to be considered contextual knowledge. Due to the dynamic nature of the current context, knowledge changes all the time. The information system able to deal with this dynamics and present it in a fast and comprehensive way is also a measure of maturity of emergency response teams.

Dissemination is very important for professionals to obtain the information necessary for the response operations. Effective disseminations transmit good quality information to all individuals involved in the response (Quarantelli 1997). In addition, supporting collaborative decision making is another challenge in response operations. The complexity of these operations often imposes a large cognitive load on the professionals involved, and can hamper decision making. Thus, information filtering mechanisms allowing that only information relevant to specific response operations is made available, allowing decision makers to focus on the decision process.

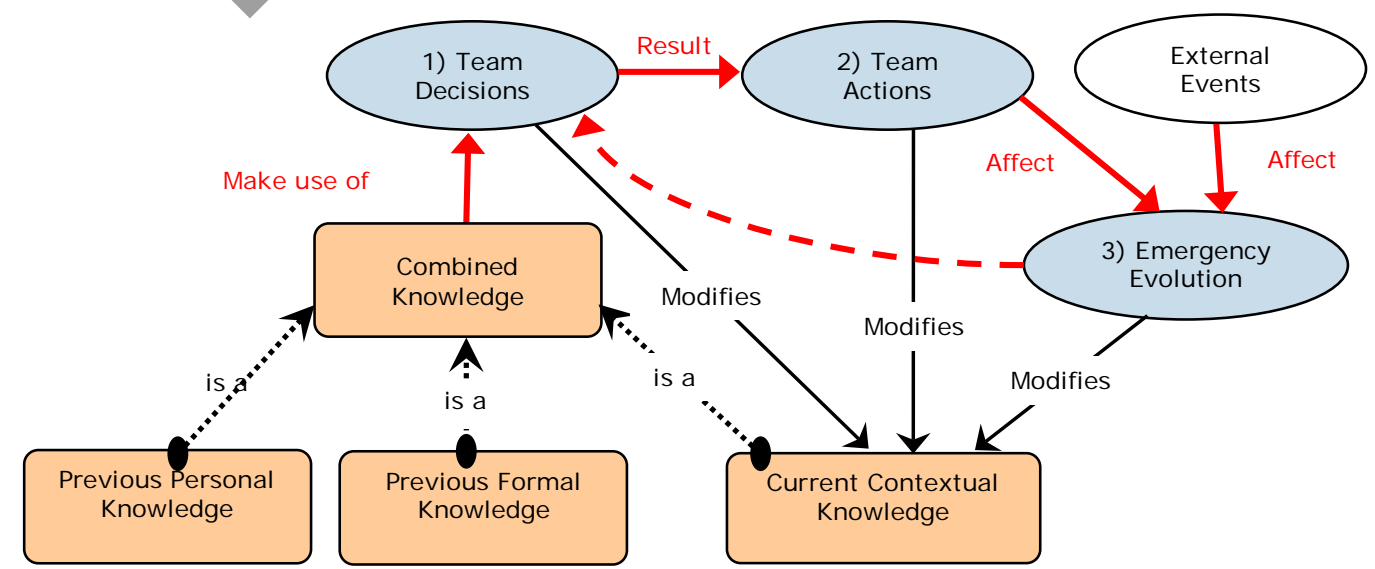

Figure 1. Knowledge Framework applied to decision making during an emergency response (Diniz et al. 2008)

Most of the knowledge managed during an emergency is generated from different information sources. In general, complex emergencies require a large amount of 
information to be gathered and processed in order to make as evidence as possible to make decisions. In other words, information must be constantly updated, disseminated in the right amount, and directed to the correct individuals (Quarantelli 1997; Palen et al. 2007). Making the right information available during emergency responses is not an easy task due to the large amount of information that may exist during this phase (Paton and Flin 1999). Thus, tool support is considered crucial to support knowledge management. Here is where Information Technologies come to the scene to play a central role via the so-called Emergency Response Management Systems.

\subsection{Emergency Response Information Systems}

Information and communication technologies (ICT) can make emergency management easier. Specifically, in the response operations, ICT can assist in capturing, representing, and disseminating the information to the professionals involved. Besides this, they can help in connecting the different organizations involved in these operations (Zimmerman and Restrepo 2006; Santos 2007). However, ICT can obstruct responses if they are not applied correctly. Information overload, under-performance, poor usability, and inappropriateness for some risk situations can be cited as problems involving ICT in emergency responses (Turoff et all 2004b).

Technology has been partially adapted to emergency scenarios (Turoff, 2002): improvements in communication networks; support for first responders by means of PDAs and intelligent cell phones and GIS-applications presenting maps that show the locations of all emergency personnel in the emergency area. Recently many works exploring mobile capabilities to support communication among emergency responders and between them and the Command and Control appeared (Ochoa et al. 2007; Yuan and Deltor 2005; Lachner and Hellwagner 2008).

Information systems are of particular importance since emergency response is an information-intensive task. Among the numerous systems available, we can mention the following ones:

- ETOILE (Dörner et al. 2001) is an environment used to train professionals in teamwork, organizational and individual learning in emergencies that may be considered as networked environment knowledge management tool.

- WIPER (Schoenharl et al. 2006) provides emergency planners and responders with an integrated system helping to detect possible emergencies, as well as suggest and evaluate possible courses of action to deal with an emergency.

- IMI (Van der Lee and van Vugt 2004) is a system that helps to integrate the diverse organizations that participate in emergency responses.

- MIKoBOS (Meissner et al. 2006) is a system for information exchange during emergency response operations.

- EmergencyCall ${ }^{1}$ uses complex technology models to contact people automatically in crisis scenarios. It uses web based, PDA and Smartphone technologies to notify people on the move about crisis situations.

${ }^{1}$ www.criticall.co.uk 
- Tapestry's Integrated CRS ${ }^{2}$ offers different operational models designed for various levels of crisis and responses. It supports evacuation registry, shelter management, case management, volunteer management and disaster planning.

- NEO: Agent Crisis Response ${ }^{3}$ (Yuan and Deltor 2005) is a system developed at Carnegie Mellon University for the interoperability of multi-agent systems to support an escalating noncombatant evacuation operation

As the list shows, different EMRSs support different aspects of emergencies. Despite their interesting functionality, from our point of view they have three main drawbacks (Llavador et al. 2006):

a) They focus on one or more particular aspects during the different phases of the emergency management lifecycle; however, none of them gives fulllifecycle support, which is crucial to give an integrated response to incidents taking into account the numerous phases and dimensions of the problem.

b) They are centered either on the emergency responders, who are experts on the resolution of emergencies (firefighters, police, medical services, etc.), or the autonomous entities that provide support for them (e.g. agents looking for information or synchronizing them). However, there are a large number of people involved in emergencies that do not get any support from current EMRSs, e.g. the citizens that suffer the incident or come to the incident place to support responders.

c) They are closed systems, sometimes configurable but not adaptable. By closed we mean that the organization must adapt to the EMRS instead of the ERMS to the organization. Thus, organization's information systems, data bases and other specific tools become disconnected from (and hence and inaccessible to) the EMRS; as a consequence, specific organization's safety requirements may remain uncovered. The alternative is to develop an entire, customized EMRS for each organization, which obviously is a difficult and costly task.

Future EMRSs should try to overcome these limitations, widening their scope to fully cover the lifecycle, serving to all the actors participating in or suffering an emergency, and being able to be configured and run according to the organizations' needs. The latter is a particularly important challenge, due to the huge diversity of organizations requiring emergency management support. Diversity comes from two different sources: on one hand, different types of organizations require different emergency procedures and practices; on the other hand, the technical capability of organizations will be a key factor to select the appropriate EMS. But assessing such capability is not trivial, due to the influence of different factors, as discussed in the next section.

\subsection{Assessing Organizations' Emergency Response Capability}

The main objective of an organization's evaluation is to provide knowledge about itself, so that it can, among other things, plan and decide on improvements to its activities. Emergency response organizations may be assessed on its capability to

\footnotetext{
${ }^{2}$ www.visionlink.org

${ }^{3}$ http://www.cs.cmu.edu/ softagents/tie3.html
} 
deal with certain types of emergencies. These assessments can be directed to one or several aspects of an emergency response, such as personnel, equipment, coordination capability, etc. Through the analysis of response activities, it is believed the organization can identify and develop improvements so that its response actions are more effective (Santos 2007).

However, it is observed that emergency response organizations rarely use assessment methods, and when they do, usually as a result of some inappropriate response, the methods are often superficial (Turoff et al. 2004b). This is understandable as these methods are generally limited to issues related to the organization's infrastructure, and often are not adequate for the reality of the organization's response operations. In other words, these methods focus on the final result of the response actions, rather than on how the outcome was achieved.

The use of maturity models is an interesting way to assess organizations (Van der Wiele et al. 2000). These models are divided in increasing maturity levels, and allow the organization, in addition to the evaluation, to plan how to reach more mature levels. This is possible as they position an organization relative to its performance of a given task, and so allow possible improvements to be identified.

\subsection{An introduction to maturity models}

Maturity models are based on the premises that people, organizations, functional areas, processes, etc., evolve through a process of development or growth in the direction of a more advanced maturity, going through a distinct number of levels. A level in the model is a base from which an evolution to a higher maturity level can be planned and implemented. In a typical maturity model the levels are: (1) sequential and cumulative by nature; (2) occur as a hierarchical progression that is not easily reversible; and (3) involve a broad array of structures and human and organizational activities (Lavoie \& Culbert, 1978). The sequential, cumulative, and evolutionary division of the maturity levels is especially important as it is able to parallel real, ideal, and possible situations relative to the allocation of an organization in a level, taking into consideration its resources and its capacity.

The concept of capability maturity has been increasingly applied to many aspects of product development, particularly in software processes (Chinowsky et al. 2007). The best known model for this purpose is the Capability Maturity Model (CMM) (Paulk et al. 1995). Originally, capability models were associated to quality assessment. More recently, the concept has been applied to assess other aspects of the organizations.

Nowadays there are various maturity models that are applied in the most varied domains. These models are applied by specialized institutes, responsible for issuing certificates according to their evaluation relative to the model considered. These institutes train professionals to perform the evaluation, and also to conduct the deployment of improvements so that the organizations can attain their maturity.

However, a very narrow application of the maturity models may become a straight-jacket and unduly restrict an organization's actions. This is particularly relevant and critical to organizations that deal with emergencies, as they have highly dynamic activities. The model must also be carefully applied to organizations whose activities are not all predefined, and often require on-the-fly adaptations to their work processes. 
In the remainder of this paper we introduce a maturity-driven model whose purpose is the assessment of emergency organization's response activities in a structured way. The use of the model can make organizations better understand their actions in response activities. This way, the identification and implementation of possible improvements to the organization's response activities can be facilitated. The model is divided in increasing maturity levels, and allows the organization, in addition to the evaluation, to plan how to reach more mature levels. This is possible as they position an organization relative to its performance of a given task, and so allow possible improvements to be identified.

\section{A maturity-based model for assessing organizations dealing with emergencies}

We have defined a model to assess the response activities of an emergency organization. The model is intended to help the organization better understands its actions in response activities, through the visualization of its knowledge and skills about these activities. This way, the identification and implementation of possible improvements to the organization's response activities can be achieved with methodological guidance.

As mentioned, maturity models allow to assess organizations in terms of increasing maturity levels. Such an assessment can be used just for evaluation purposes, but it can also include the actions to reach a higher maturity level.

The organization is analyzed from the point of view of different dimensions related to emergency response activities (see section 2.1), which can be considered jointly or separately. The analysis can be made by independent specialists or groups, each one in charge of a specific dimension; for each dimension, an evaluation is made. Additionally, an overall evaluation of the organization, combining the different assessments into a single framework, can be produced as the result of the collaboration of experts.

The model divides each dimension into maturity levels. Each level has requirements that must be complied with by an organization to be allocated to it. The analysis tries to assess the degree of fulfillment of such requirements. Being aware of its maturity in the different dimensions, the organization comes to know the points to prioritize in order to reach higher maturity levels.
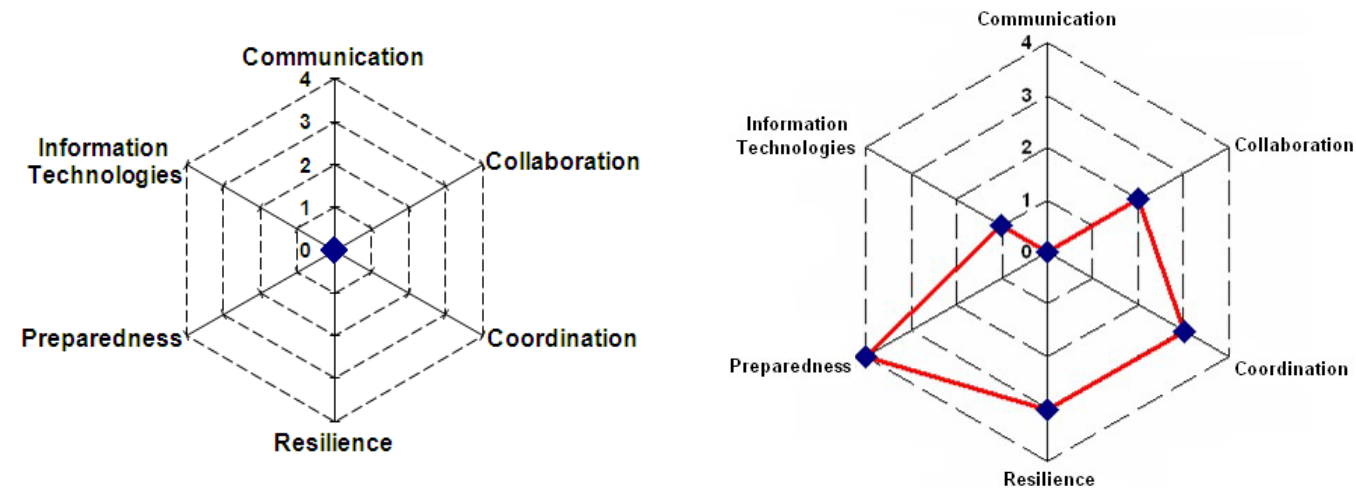

Figure 2a,b: Radar graph examples. Dimensions and assessment results

Given the high number of dimensions, with different maturity levels in each one, we needed a notation that allows visualizing the assessments in a clear and (if 
possible) concise way. We selected Radar Graphs as they afford a multidimensional view of the model, showing each dimension separately or grouped into a higher-level dimension. Figure 2. a shows the radar graph we use to represent the organization at the highest level of abstraction. It has an edge for each dimension considered. For each edge, a number of maturity levels have been defined. Figure 2.b shows the assessment made of a specific organization. Looking at the diagram, the organization may decide to invest to increase its maturity in terms of communication (rated 0 in its current evaluation), whereas it may feel satisfied with the preparedness level (rated the highest in the scale).

The rating of the organization in each dimension is obtained from the combination of values of lower-level properties, as we illustrate in our case study (see Section 4). The way in which different values are combined is organization-dependant, and selecting the criteria requires expertise on the domain.

As response activities vary among domains, the first challenge is to find the dimensions for a particular case. As a consequence, some guidance to build a model for each domain is required (Santos 2007).

\subsection{A method for building maturity models}

A domain-specific maturity model must be developed carefully. Model engineers have to acquire a detailed knowledge of the domain, either by themselves or with the help of experts in the domain. This knowledge must be organized into a number of dimensions, for each of which a maturity scale must be defined. The resulting model must be reviewed by the experts, resulting in possible improvements in both dimensions and maturity scales. The maturity model construction process is depicted in Figure 3. It is an iterative process consisting of a number of stages that we describe in detail below.

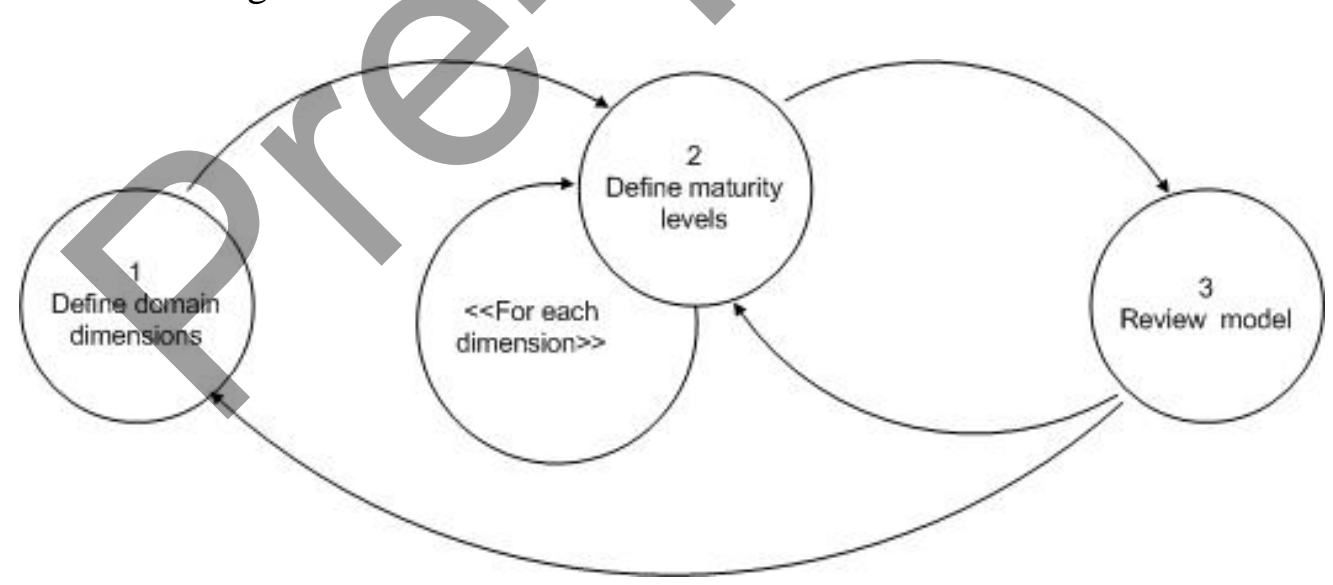

Fig 3: The maturity level definition process

\subsubsection{Definition of the dimensions related to the domain's response activities.}

The first step is the description of the component structure that will constitute the model for the domain. The structure must be defined in such a way that it can faithfully portray the response activities carried out by the emergency organizations belonging to the domain. Since analyzing the organization relative to each dimension is not trivial, we use decomposition based techniques to ease the analysis. At each level, dimensions are divided into sub-components (lowerlevel dimensions) whose analysis is less complex; the decomposition should go on 
until components are obtained whose assessment is possible through direct observation and/or measurement of the organization's variables.

Each component of the model belongs to a given level of abstraction. A level of abstraction may have " $m$ " elements for which the assessment of an organization's response capacity has similar complexity. The levels of abstraction are organized hierarchically, being the greater ones, that is, those with more complex assessment, are at the top of the hierarchy (see Figure 4).

The number of abstraction layers will depend on the domain: there should be as many as necessary to reach a level of abstraction in which the organization's assessment can be obtained. Notice that levels of abstraction and maturity levels are not the same: the former determine what should be observed to assess the organizations, whereas the latter correspond to the ranking of the elements at one given level of abstraction.

As mentioned, the definition of the component structure should be guided by experts in the domain for which the model will be built. It is recommendable that more than one specialist helps to create the definition, as different points of view can bring the component structure closer to the reality of emergency response organizations' operations in the domain.

\subsubsection{Definition of the model's maturity levels}

The second step in the method consists of defining the maturity levels for every dimension at each abstraction level of the model. Each maturity level is defined in terms of the characteristics that an organization must fulfill to be deemed at that level.

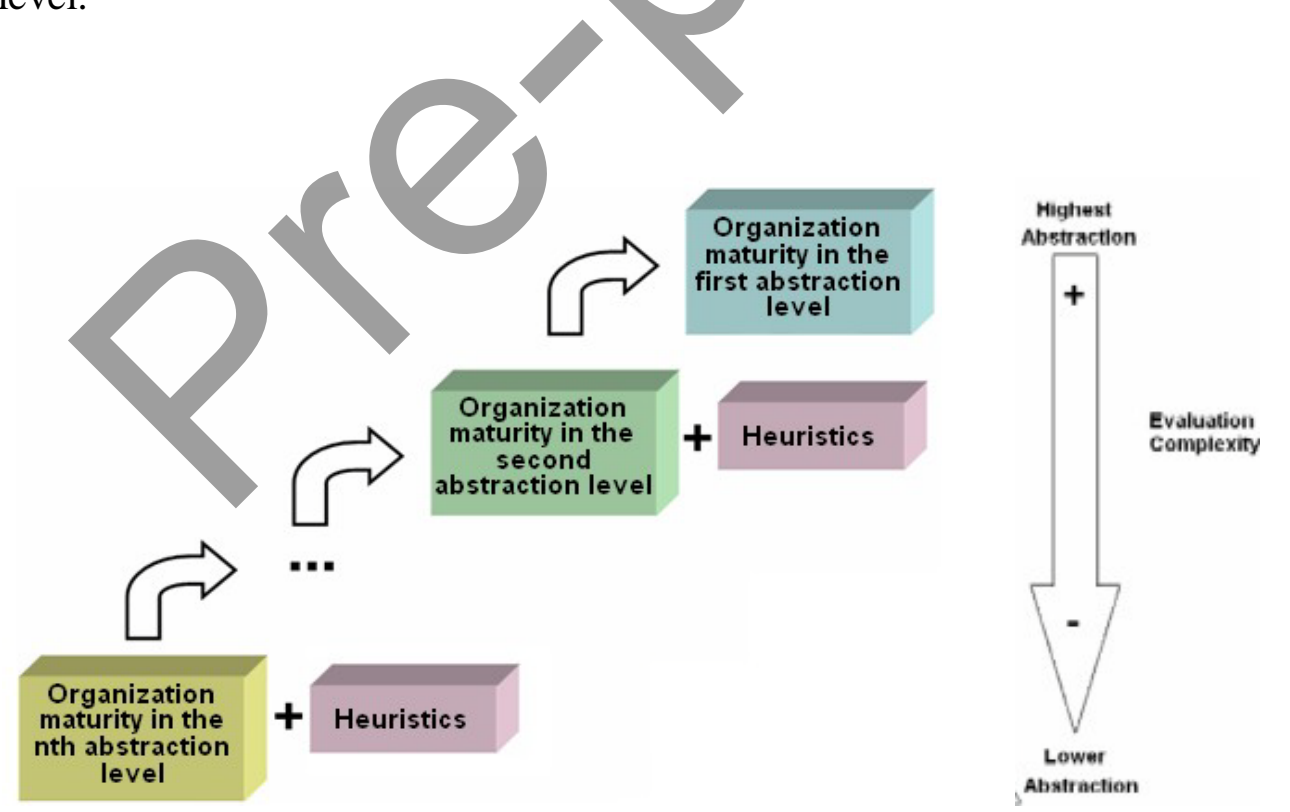

Figure 4: Assessing the organization’s capability using the maturity levels [Santos et al., 2008].

Figure 4 shows how an emergency organization's maturity is evaluated using the model. Following a bottom-up strategy, the maturity of the organization relative to the elements of the lowest level of abstraction is obtained first. After this classification, heuristics are applied to the maturity ratings obtained, giving rise to the organization's maturity values relative to the elements of the abstraction immediately upper level. The application of heuristics is repeated until the 
organization's maturity relative to the elements of the highest level of abstraction is reached.

Once situated at a maturity level relative to an element of the levels of abstraction, the organization comes to know its negative and positive points regarding its emergency response operations. Additionally, the organization can identify procedures that will allow it to overcome its negative points. As the organization overcomes its negative points, it increases its maturity level in the model's hierarchy, that is, it increases its response capacity. Ideally, the organization manages to make itself mature (reach the highest maturity level) in all of the model's components, gradually and evenly.

\subsubsection{Model review}

The third and last step of the method proposed here is the review of the model built. No matter how perfect the model built, little details may not represent emergency response operations faithfully. Reviewing is necessary for these details to be fixed. Domain specialists are interviewed so that an analysis of the model's items is done. This analysis happens through comparisons with the response operations of emergency organizations in the domain, from the interviewees' point-of-view.

During the review process, any consideration about the model constructed can be made, and if possible, registered. The pertinent considerations must be implemented until the resultant model is considered able to be applied in assessing emergency organizations' response capacity, producing results compatible with the organization's performance in response operations.

\section{A case study: applying the method to a fire- fighting organization}

We illustrate the main characteristics of our method by applying it to the case of fire-fighting organizations. This type of organization is particularly relevant in emergency response, as its members are involved in numerous tasks requiring expertise, equipment and high preparedness.

\subsection{Definition of the model}

To build the maturity model, a detailed analysis of the domain was performed to define the hierarchy of abstraction levels in a way compatible with the domain organization's operating reality. The analysis consisted of interviews with domain specialists and studies undertaken about the domain. As a result, we initially found the need for three levels of abstraction for the model. This conclusion followed from observations as well as from reports found in the literature and obtained in the interviews (Turoff et al. 2004).

Consequently, we defined a three-level hierarchy of abstraction levels, on top of which we placed the so-called indicators, which correspond to the dimensions present in the emergency response operations of firefighters, such as communication, collaboration, coordination, information technologies, information management, resilience, preparedness, etc. Additionally, other 
indicators may be identified in future domain analyses, due to different knowledge and points of view among the professionals responsible for these analyses.

At the second level we placed the criteria; a criterion is a dimension subordinated to an indicator, which may be in turn decomposed into aspects, which are the components at the lowest abstraction level. In this paper, we focus on the study we performed about the "Information Technologies" indicator, since information management is one of the most important elements present in response activities.

Information technologies must comply with a number of requirements to be effectively used. The requirements are those relative to information capturing, storage, dissemination, and quality. Despite their relevance, information technologies are often used without a comprehensive analysis of these requirements. In such cases, information technologies do not satisfy the needs for which they are employed, which may indicate wasted resources, a critical issue in places where investments are scarce.

The model allows the visualization of how a fire-fighting organization's information technologies perform relative to their requirements. Such visualization is possible as the model was developed to assign priority to the requirements related to information technologies. After the study in the firefighting organization domain, and taking into account the concepts related to information technologies in the responses, the criteria that compose the selected indicator were defined. These criteria do not take into account specific technologies, but rather the functionalities they are able to provide. The criteria defined for this indicator are described below and summarized in Fig. 1.

Information Capture: how the organization's information technologies are used to capture information useful for emergency responses? It is expected that this criterion will facilitate the organization's planning of measures seeking to improve the use of information technologies to capture information for emergency responses. The aspects verified for this criterion are:

- The policies and strategies to stimulate the use of information technologies to capture information for emergency responses;

- The techniques employed to capture information for emergency responses;

- Information filtering while capturing;

Information Storage: how does the organization use technologies to store information captured for emergency responses? It will be possible to assist the organization in planning improvements related to the storage of its information, and to a certain degree, foresee what its information dissemination may be like. The aspects verified for this criterion are:

- The bases for the storage of the information captured for emergency responses;

- The standardization of the information captured for emergency responses.

Information Dissemination: which technologies are used for the dissemination of information during emergency responses? It is important that during the response activities, the right information be transmitted to the right people, at the right time. Thus, it may be possible to assist the organization in planning improvements 
related to the dissemination of its information. The aspects verified for this criterion are:

- The importance of the disseminated information to the emergency responses;

- The reach of the dissemination of information for emergency responses;

- The availability of the disseminated information to the emergency responders;

- The filtering of information during dissemination;

- The use of disseminated information in emergency responses.

Information Quality: what is the quality of emergency response related information which is managed by the information technologies used by the organization? We considered quality information that is relevant for a given emergency response. This criterion enables an organization to improve the quality of its response related information, and consequently the outcome of its response activities. The aspects verified for this criterion are:

- The relevance of the information to emergency responses;

- The accuracy of the information for emergency responses.

Inter-Organizational Information: how the organization's information technologies deal with inter-organizational information during emergency responses? The importance of this criterion is justified as this type of information circulates during most emergency response activities. The aspects verified for this criterion are:

- The policies adopted to promote getting and exchanging inter-organizational information during emergency responses with information technology support;

- The strategies used to promote getting and exchanging inter-organizational information during emergency responses with information technology support;

- The tactics used to promote getting and exchanging inter-organizational information during emergency responses with information technology support;

- The technological infrastructure for getting and exchanging inter-organizational information during emergency responses;

- The ability for inter-organizational communication.

Previous Information: how previously formalized information is treated by the organization's information technologies, for using during emergency responses? This type of information includes consolidated concepts and information extracted from previous events, deemed important for future emergencies. Since it is related to future events, we believe that with this criterion the organization will be able to better prepare its professionals for possible emergency responses. The aspects verified for this criterion are:

- The formalization of previous information for emergency responses;

- The use of previous information in emergency responses;

- The dissemination of previous information for emergency responses;

- The previous inter-organizational information for emergency responses. 


\subsection{Model evaluation}

Once the model was built, it was necessary to check with specialists whether it was consistent with the response operations in the domain or not. The first version of the model was generated by non-domain specialists. This version was given to three specialists with different knowledge of response operations, who performed the critical review of the model. They were asked to analyze the division of the model's components and the maturity level structure, indicating and suggesting changes and improvements so that the modeled indicator became as consistent as possible with the reality of the response operations in the domain. According to the specialists, the final model faithfully portrays the response activities in the fire-fighting domain, and can therefore be used in the assessment of organizations.

\subsection{Application of the model}

For the first application, we selected the Firefighters Department of the State of Rio de Janeiro. Initially, a study of the organization was done, conducted mostly through interviews with the organization's professionals with the intent of adapting the model's application to the organization's characteristics. The study permitted the identification and the understanding of the organization's structure, which contributed to its assessment within the framework defined by the model.

The assessment was conducted through interview sessions supported by a tool implemented in a Microsoft Excel spreadsheet using VBA (Visual Basic for Applications) macros. There was a separate session for each of the interviewees. With the aim of facilitating the assessment task, a presentation before each session showed how the assessment would be conducted (specifically, the model's multilevel structure concepts) and the expected results. The presentation also included a short tutorial about the use of the tool.

The tool's spreadsheet was conceived to facilitate data collection and graphically represent the organization's maturity. From the data supplied by the interviewees, the spreadsheet automatically generates graphs representing the organization's maturity regarding its emergency response activities. Initially, the user must include the values for the aspects of the lowest level criteria associated to a dimension. The process had to be repeated for every dimension.

To move up in the abstraction level hierarchy, the user must select one of the predefined heuristics, or define a new one. A heuristic is a function defined by the domain specialist for combining the lower level values into a value at the upper level. For instance, if the lower level values are 3, 2 and 1, the average function will give the value 2 at the upper level. A minimum function would give the value 1 and so on. The two predefined heuristics are: simple average, and weighted average of the maturity lower levels. After the heuristics of different levels have been selected, the tool applies the calculations relative to the different heuristics in the appropriate order to construct the organization's maturity evaluation.

The tool synthesized the data collected into graphs. With them, it became easy to identify which were the organization's positive and negative points regarding its emergency response activities. Once these points were exposed, it was possible to identify and recommend improvements to the organization's response capability. The identification also enabled a comparison between the evaluated units.

We analyzed three operational units of the organization. In the remainder of this section, we show the results for the assessment of two of them, omitting the third 
one's due to lack of space. The evaluation was obtained using the weighted average of maturity levels. The weight of each of the model's components was defined by consensus. A unique heuristic function for all three units enabled a straight comparison of the three units' assessments.

\section{Unit A}

The first unit evaluated serves a relatively small area where the frequency of incident occurrences is low. The unit has on average 100 firefighters, divided into shifts, performing the various duties to assure success of the emergency responses. To evaluate its maturity level, one unit's officer participated in the evaluation process.

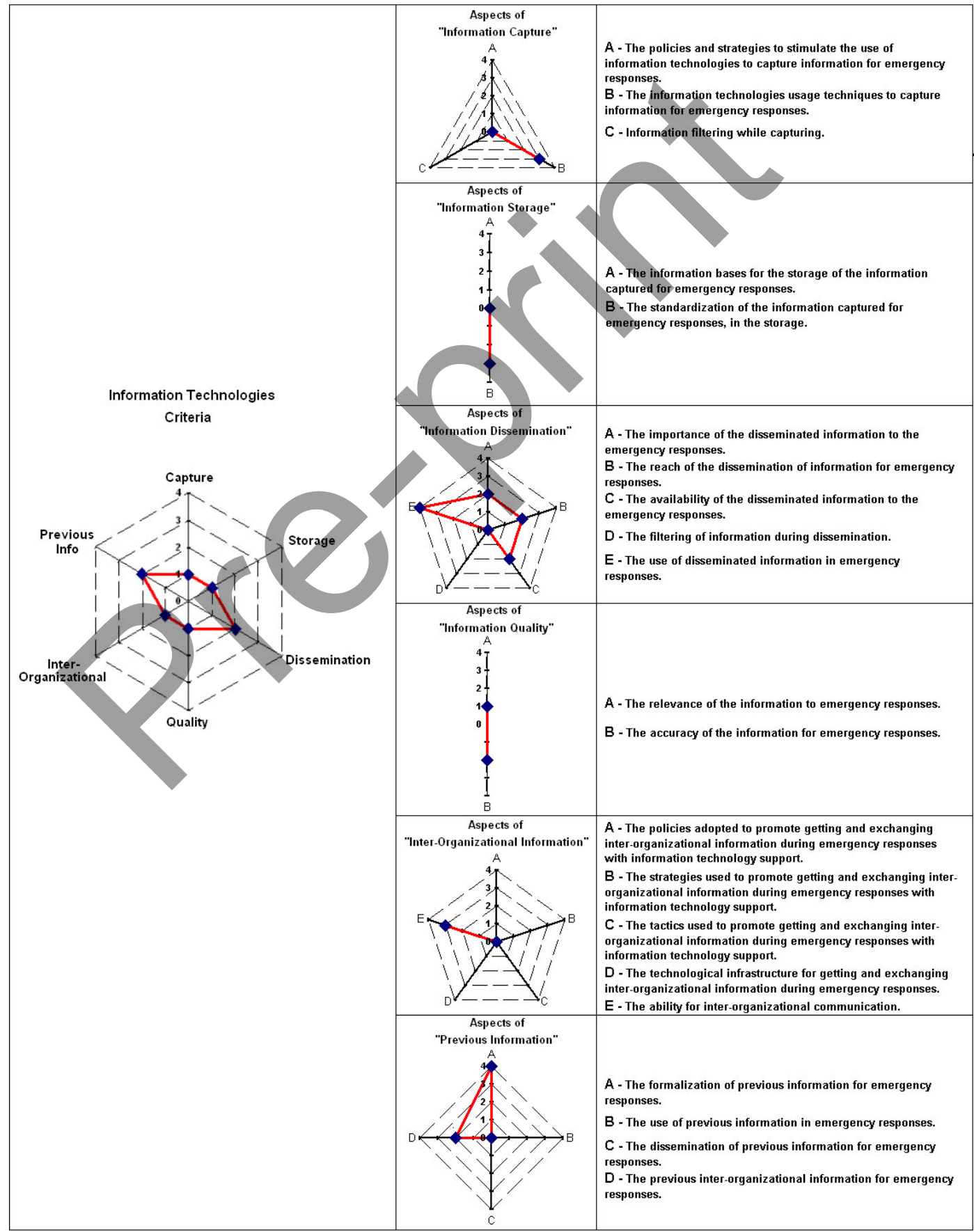

Figure 5: Unit A's maturity levels relative to the criteria and aspects of the "Information Technology" indicator 
The assessment showed that unit $\mathrm{A}$ is in the first maturity level relative to information technologies. This means that its use of information technologies is still ad hoc. This was not a uniform result: in some criteria the unit showed satisfactory responses, whereas it showed problems in others. Figure 5 shows the graphs that indicate the unit's maturity levels relative to the criteria and the aspects that make up the "Information Technology" indicator. According to the graphs, the unit makes best use of information technology in dissemination and in treating previous information. In contrast, its use of information technologies is not very effective relative to the capture and storage of emergency response activity information, and shows little concern with the quality of response information and with inter-unit information.

Although the organization has in place relatively effective measures for information dissemination during responses, the amount is not high because the unit has not systematized capture and storage procedures. Additionally, the response information lacks quality, and may impair the response activities.

The interviewed unit's officer considered the assessment method to be simple and very useful for the unit. According to him, the method got close to the reality of the unit's response operations, providing a better visualization of the knowledge related to the use of information technology in the response activities and facilitating the identification of positive and negative points of the organization in these activities. On the other hand, he criticized the way in which the assessment was conducted. According to him, other professionals should have participated in the assessment. Only one professional participating in the assessment may skew the results. Also, the interviewee concluded that the assessment should be carried out during a longer period, instead of only one interview.

\section{Unit B}

The second unit evaluated serves an area larger than unit A that presents a larger number of events. This may be caused by the area's high population density, which may affect emergency-causing factors such as in-traffic, types of housing, commercial and industrial installations, etc. Consequently, the manpower of the Unit is greater, as well as its infrastructure, in terms of both installations and equipment.

The study concluded that Unit B, like the unit A, is at the first maturity level relative to the "Information Technologies" indicator. However, the reasons for this classification are different. The graphs shown in Figure 6 show the unit's information technologies are used efficiently for the storage of emergency response activity information. They also indicate that the unit uses its information technologies to capture and disseminate quality information during its emergency response operations.

The decomposition of each indicator in less-complex components, according to the unit B's interviewee, allowed the identification of factors that he did not imagine could influence the organization's response performance. These factors included the systematization of the use of technologies to treat interorganizational information, and the use of information technologies to support the formalization of previous information. As a drawback of the method, the interviewee mentioned the need to consult professionals more familiar with certain response activities. 


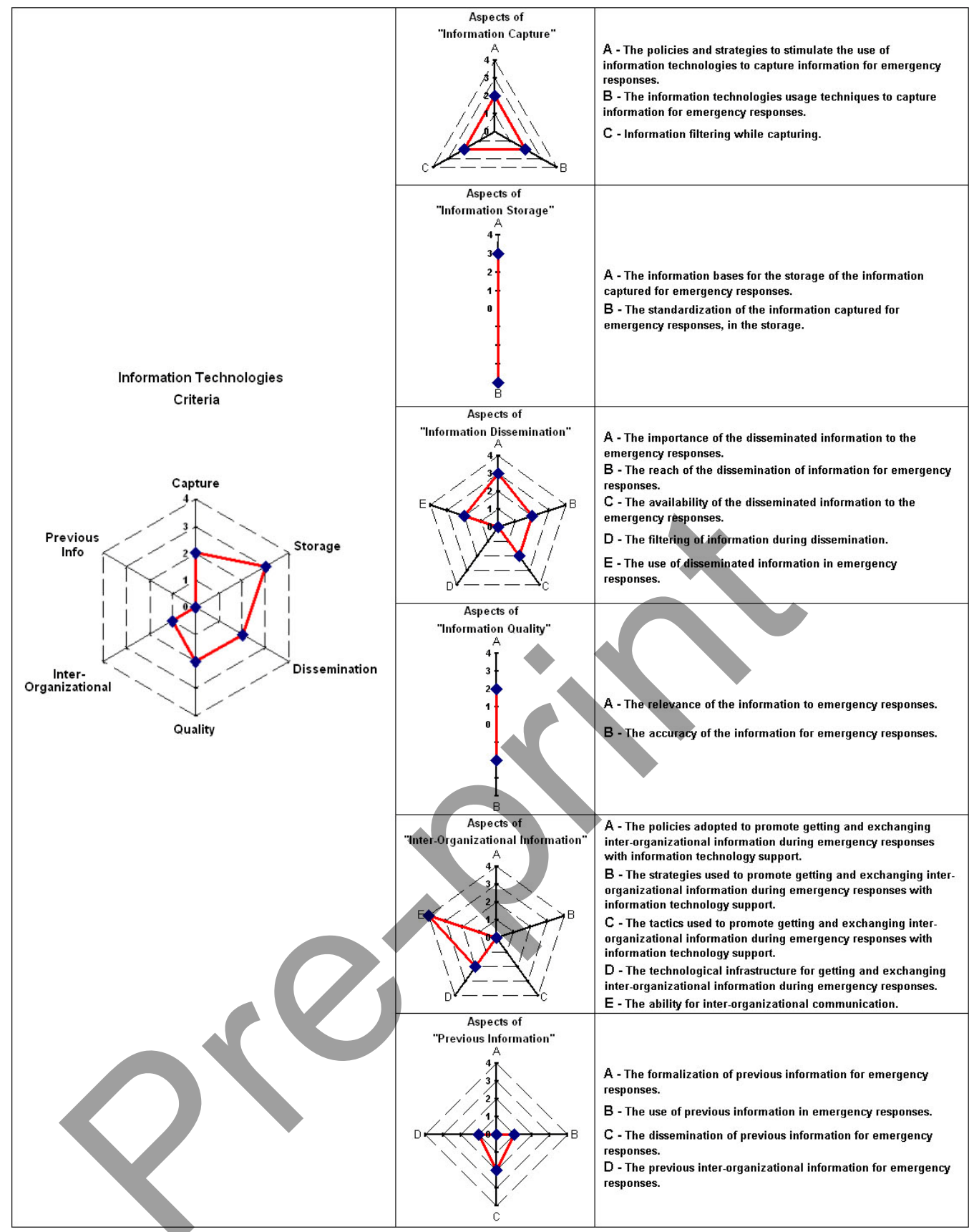

Figure 6: Unit B's maturity levels relative to the criteria and aspects of the "Information Technology" indicator

\section{Conclusions}

The solution presented here began from an important issue: an emergency organization's knowledge of how it operates in the course of its response activities. It is difficult for the organization to satisfactorily understand how it is prepared to respond to emergency situations. An assessment method, such as the one proposed in this article can help organizations to identify its limitations and to guide their investments on improving their capabilities. A reference model based on the maturity model concepts has been proposed to support the evaluation of the response capability of emergency organizations. 
In spite of being based on the maturity model concepts, the model proposed does not have their characteristic rigidity. This method makes the model dynamic, and can adapt it to evaluate organizations of any emergency related domain, in addition to allowing the concern of any dimension involved in possible emergencies. The method also allows the model to be extended to model the organization's capability in other phases of emergency management.

Evaluation through the model positions the organization within its emergency response activities performance spectrum, showing it a portrait of that potential performance. This portrait has the organization's positive and negative points regarding its resources to respond to emergency situations, which may more effectively guide the organization's focus in the search for improvements to these activities.

At this point we can say that there is some indication that our hypotheses hold in the development of a model for the fire-fighting domain, and its application in the Fire-Fighting Departments. The model was developed with only one dimension: the information technologies. In it some issues related to these technologies in this specific domain's response activities were raised and detailed.

Although specialists did not develop the entire assessment, participants praised its proximity to the organization's response operations reality. This proximity produced results coherent with the current use of information technologies by the organization in emergency responses. The participants praised the model and reported the importance of an assessment that translates the organization's reality. According to them, the results translated clearly the organization's real needs regarding information technologies for emergency responses.

An assessment method allows for an organization to know itself. A specific assessment model developed for the environment where the organization is situated promotes a better understanding of what is valuable to the organization. With the model and the results of its assessment an organization should be able to identify its weaknesses and strengths and to address them. In the organizations mentioned in the case study, the managers used the assessment reports to support their request for investments in their weak aspects. They also offered help to other organizations on the aspects in which they are strong.

When the model and the assessment is applied to several organizations of the same type, it generates a benchmark process. By understanding and making explicit their weaknesses and strengths, organizations can create a collaborative environment promoting exchange of expertise and joining efforts to deal with deficiencies which are common to all or most of them.

A variant of the method and the model has been developed and applied to the assessment of the risks pertaining to infrastructure in an oil company in Brazil. The model and the method have been adopted to define a strategic action plan in a way to balance investments adequately in order to achieve a steady reduction of risk. The company is not an emergency response organization, but it also uses the information technology as its measurable target. The full model developed for this assessment has 9 indicators, which, on average, have 4 criteria each. Each of these criteria has 4 aspects on average, some of them with a further level. On the total, over 300 hundred aspects were evaluated. The exercise has demonstrated a broader usage of the maturity model. 
A third application is under way. A more refined maturity model is under construction to serve as a way to assess educational units. The model started with three dimensions and over a hundred aspects, but it is rapidly growing. It will be initially used to assess private colleges and will try to suggest where to apply resources to improve the quality of these institutions.

\section{Acknowledgements}

Marcos R. S. Borges was partially supported by grants No. 304252/2008-5 and 480461/2009-0 from CNPq (Brazil). Work of José H. Canós is partially funded by the Spanish Government through the grant DEEPEN (TIN2008-08084) and the Junta de Comunidades de Castilla-La Mancha under project INGENIO (PAC08-0154-9262). The cooperation between the Brazilian and the Spanish research groups was partially sponsored by the CAPES/MECD Cooperation Program, Project \#169/ PHB2007-0064-PC.

\section{Bibliography}

Bigley, G. A., Roberts, K. H.: The incident command system: high reliability organizing for complex and volatile task environments. Academy of Management Journal 44 (6), 1281-1299 (2001)

Chinowsky, P., Molenaar, K. and Realph, A. Learning Organizations in Construction, Journal of Management in Engineering, Volume 23, Issue 1, pp. 27-34 (2007)

Diniz, V. B., Borges, M.R.S., Gomes, J.O., Canós, J.H.: Decision Making Support in Emergency Response. In: Encyclopedia of Decision Making, pp. 184-191. Information Science Reference (an imprint of IGI Global), New York (2008)

Dörner, R., Grimm, P., Seiler, C.: ETOILE - An Environment for Team, Organizational and Individual Learning, CG Topics 13 (3), 5-6 (2001)

Dykstra, E. "Concept paper: Toward an international system model in emergency management". Proceedings of Toward an International System Model in Emergency Management, Public Entity Risk Institute (2003)

Federal Emergency Management Agency (FEMA): Emergency Management Guide for Business and Industry: A Step-by-step Approach to Emergency Planning, Response and Recovery for Companies of All Sizes (1998)

Gu, Q., Mendonça, D.: Patterns of group information seeking in a simulated emergency response environment. In: Proceedings of the $2^{\text {nd }}$ International ISCRAM Conference, Brussels, Belgium (2005)

Hale, J.: A layered communication architecture for the support of crisis response. Journal of Management Information Systems 14 (1), 235-255 (1997)

King, W., Teo, T.: Integration between Business Planning and Information Systems Planning: Validating a Stage Hypothesis. Decision Sciences 28 (2), 279-307 (1997)

Lachner, J. and Hellwagner, H.: Information and Communication Systems for Mobile Emergency Response, Lecture Notes in Business Information Processing, Vol 5, p. 213-224 (2008).

Lavoie, D. and Culbert, A.: Stages in organization and development, Human Relations, $\mathrm{n}^{\mathrm{o}}$ 31, p. 417-438 (1978)

Lindel, M. K., Prater, C., Perry, R. W.: Emergency Management. John Wiley \& Sons, New York (2007)

Llavador, M., Letelier, P., Penadés, M.C., Borges, M.R.S., Solís, C.: Precise yet Flexible Specification of Emergency Resolution Procedures. Proceedings of the Information Systems for Crisis Response and Management (ISCRAM) p. 110-120 (2006)

Meissner, A., Wang, Z., Putz, W., Grimmer, J.: MIKoBOS: A mobile information and communication system for emergency response. In: Proceedings of the $3^{\text {rd }}$ International ISCRAM Conference, Newark, New Jersey (2006) 
Nonaka, I., \& Takeuchi, H. (1995). The knowledge creating company: How Japanese companies create the dynamics of innovation. Oxford University Press , Oxford (1995)

Ochoa, S., Neyem, A., Pino, J.A., \& Borges, M.R.S. Supporting group decision making and coordination in urban disasters relief efforts. Journal of Decision Systems, 16(2), 143-172 (2007)

Paton, D., Flin, R.: Disaster stress: an emergency management perspective. Disaster Prevention and Management 8 (4), 261-267 (1999)

Paulk, M.C., Weber, C., Curtis, B., Chrissis, M.: The Capability Maturity Model: guidelines for improving the software process. Addison-Wesley, Reading (1995)

Quarantelli, E. L.: Problematical aspects of the information / communication revolution for disaster planning and research: ten non-technical issues and questions. Disaster Prevention and Management 6 (2), 94-106 (1997)

Santos, R. S., Borges, M.R.S., Gomes, J.O., Canós, J.H.: Maturity Levels of Information Technologies in Emergency Response Organizations. In: Proceedings of the International Workshop on Groupware, Omaha, Nebraska, USA. Groupware: Design, Implementation and Use Lecture Notes in Computer Science. Berlin, Heidelberg : Springer, v. 5411, p. 135-150 (2008)

Schoenharl, T., Szabo, G., Madey, G., Barabasi, A. L.: WIPER: A multi-agent system for emergency response. In: Proceedings of the $3^{\text {rd }}$ International ISCRAM Conference, Newark, New Jersey (2006)

Turoff, M.: Past and Future Emergency Response Information Systems. Communications of the ACM 45 (4), 29-33 (2002)

Turoff, M., Chumer, M., Hiltz, R., Clasher, R., Alles, M., Vasarhelyi, M., Kogan, A.: Assuring Homeland Security: Continuous Monitoring, Control and Assurance of Emergency Preparedness. Journal of Information Technology Theory and Application (JITTA) 6 (3), 1-24 (2004a)

Turoff, M., Chumer, M., Van de Walle, B. and Yao, X. The design of a dynamic emergency response management information system (DERMIS), Journal of Information Technology Theory and Application (JITTA), 5:4, p. 1-35 (2004b)

Van der Lee, M. D. E., Van Vugt, M.: IMI - An information system for effective multidisciplinary incident management. In: Proceedings of the $1^{\text {st }}$ International ISCRAM Conference, Brussels, Belgium. (2004)

Yuan, Y., Deltor, B. "Intelligent Mobile Crisis Response Systems", Communications of the ACM,.28(2) pp. 95-98 (2005)

Zimmerman, R., Restrepo, C. E.: Information technology (IT) and critical infrastructure interdependencies for emergency response. In: Proceedings of the $3^{\text {rd }}$ International ISCRAM Conference, Newark, New Jersey (2006) 